\title{
¿BURNOUT? UN ESTUDIO A UN GRUPO DE DOCENTES DE LA CARRERA DE FINANZAS Y AUDITORIA
}

\section{¿BURNOUT? A RESEARCH IN A GROUP OF PROFESSORS OF THE FINANCE AND AUDIT CAREER}

\author{
Maria Cristina Araque Salazarl, William Marcelo Moreno Cazar²
}

\begin{abstract}
Resumen
El estudio analiza el síndrome de BURNOUT en docentes con dedicación medio tiempo y tiempo completo en la carrera de Finanzas y Auditoría, con el objetivo de identificar la existencia de este factor psicosocial y las evidencias en relación con las manifestaciones características tanto en el humor como en la salud de los encuestados, relacionadas con las horas laboradas y el tiempo destinado a su vida familiar y personal. Es decir, existe relación entre el tipo de trabajo y la incidencia del sindrome de BURNOUT, para lo cual se emplearon encuestas anónimas a los docentes. Se utiliza un modelo descriptivo, a través del cual se obtuvieron los datos para determinar las caracteristicas particulares de cada docente, posibles cambios emocionales o sintomatología. Para esto, se aplicó una encuesta con preguntas cerradas derivadas del marco teórico, donde se puede identificar que la docencia y el contacto constante con las necesidades y requerimientos de los estudiantes, sumado a la exigencia académica, a la gestión docente y a la investigación, inevitablemente restan tiempo a la vida personal y familiar de los académicos. La encuesta confirma el problema planteado respecto a la presunción de vulnerabilidad a este riesgo psicosocial. Los resultados arrojaron datos sobre la afectación de los docentes en cuanto a su salud física y emocional que corroboran el planteamiento de a mayor exigencia laboral más síntomas y dolencias.
\end{abstract}

\section{Palabras Claves}

Enfermedad en el trabajo, docencia, seguridad laboral, salud mental, riesgo psicosocial.

Clasificación JEL: J28/ Seguridad; Accidentes; Salud industrial; Satisfacción en el trabajo; Política Pública Relacionada.

\begin{abstract}
This research analyses regarding the BURNOUT syndrome in a group of university professors hired part time and full time in the Finance and Audit Career, with the objective of identifying the existence of this psychosocial factor and the evidence in relation to the changes in their mood or health is was related to their working hours and the lack of time assigned to their family and personal life. Therefore, the study shows a relationship between the type of work university school engages and the burnout effects that can be considered psycho-social issue, anonymous surveys were conducted in order to research the variables involved in this problem, they are the combination of teaching, regular contact with students in order to support their educational needs, new added academic requirements additional, administrative and management tasks and research, which in sum take time away from the personal and family life of these faculty members.
\end{abstract}

A descriptive analysis is proposed throughout this study that provides data about the particular characteristics of each professor, about their changes of emotional changes and/or symptomatology, where it was evidenced that the administration of personal and family time depended directly on tasks and responsibilities assumed with them. The results give data on the involvement of teachers in terms of their physical and emotional health that corroborate the approach to greater labor requirement more symptoms and ailments.

\section{Keywords}

Work illness, teaching, job security, mental health, psychosocial risk.

JEL Classification: J28 / Safety; Job Satisfaction; Related Public Policy 


\section{Introducción}

El síndrome de burnout es el desgaste emocional, físico y psicológico que se presenta frente a las crecientes demandas del entorno laboral y la carga de trabajo y se traduce en la forma en la que afectan al individuo generando sentimientos de insatisfacción y desaliento limitando su realización profesional y personal dentro de sus funciones (Pérez, Angulo, Cruz y Estrada, 2018).

El estudio del síndrome de burnout parte desde el año 1971, con la conceptualización del estrés, a pesar de la polémica que existe por sus definiciones de tipo teórico hasta el desarrollo del tema en cuestión, ya que existe de por medio una predisposición individual a sentir los efectos que produce esta, transformándola netamente en un "modelo multidimensional" con una repercusión de "contexto social" (Arquero y Donoso, 2013).

La directriz predominante de servicio a los demás que tiene la docencia, termina traduciéndose en un detrimento de la salud física y emocional debido al contexto propio que enfrentan las instituciones de educación superior en el Ecuador estos momentos, donde los indicadores de calidad y cumplimiento deben ser revisados constantemente (Romero, Pimienta, Ramos, Sanchez y González, 2019).

La salud laboral ha sido constante objeto de estudio y son los factores psicosociales es uno de los riesgos que no solo producen enfermedades profesionales, sino también, influyen en el estado cognitivo, emocional y conductual de los trabajadores. Dentro de este grupo se identifican a los docentes que se encuentran sometidos a exigencias laborales en cuanto a plazos, volumen de trabajo, demandas de estudiantes con escasa preparación, poco colaboradores

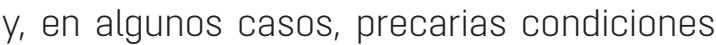
de trabajo (Serpa, 2013). Las enfermedades que presentan los trabajadores sometidos a altos niveles de estrés son trastornos gastrointestinales, respiratorios, musculares, entre otros, que aunados a padecimientos de ansiedad y depresión terminan por "quemar" a los profesionales (Granados, Aparisi, Inglés, Aparicio, Fernández y Garcia 2019).

La normativa legal en términos de Seguridad y Salud Ocupacional determina el estudio exhaustivo de los riesgos psicosociales a los que se encuentran sometidos los trabajadores; uno de estos es el "proceso de desgaste profesional" mejor conocido como burnout que en el contexto psicosocial se define como un síndrome, en el que se combinan el medio laboral y la individualidad de los trabajadores, ya que comprende "sentimientos y conductas" existiendo una marcada predisposición en aquellos que trabajan en un relacionamiento humano como son los docentes, entre otros (Carlin y Garcés, 2010).

Según el análisis realizado por Carlotto, Mary Sandra, Nakamura Antonieta Pepe, (como se citó por Freire et al., 2015) existen "las tres dimensiones del síndrome de burnout son: el agotamiento emocional, la despersonalización y la realización profesional". La primera considera el desaliento y las escasas expectativas; la segunda trata de la carencia de empatía con los que se relaciona, y la tercera habla del deterioro de la competitividad laboral. Cada uno de estos factores afecta en diferente magnitud a los docentes universitarios, entendiendo la individualidad propia y la sensibilidad personal que derivan en la existencia de sintomatología de afectación laboral, incidiendo en índices de morbilidad (Freire, Oliveira, Guimarães, Lopes, Nogueira y Vieira, 2015).

De acuerdo a lo expresado por Maslach y Jackson (1981), existe una serie de factores que podrían predisponer a los individuos, en especial a los docentes, por su significativo contacto con los alumnos, sus necesidades y requerimientos, que se traducen a posterior en un desencadenante de 
la presencia de uno o más síntomas (como dolor de cabeza, espalda, trastornos de sueño, fatiga crónica, irritabilidad, pérdida de memoria, migrañas, síntomas de depresión, ataques de pánico, entre otros). Prevalecen algunos estudios en los que se entiende que la desproporción del tiempo destinado a las labores y las continuas exigencias a las que se someten los trabajadores terminan convirtiéndose en una de las causas más significativas de incapacidad laboral y también de morbilidad psiquiátrica ocasionadas por el estrés (Sauter, Murphy, Hurrell y Lennart, 2001).

Los acelerados cambios que se han producido respecto a la educación superior, se han visto reflejados en que tanto los académicos como los gobiernos estudien este fenómeno (Murphy y Adams, 1998). El panorama que enfrenta la educación en la actualidad se basa en un entorno efímero, lleno de constantes cambios y desafíos planteados por "la era de la información y de la incertidumbre", donde se busca predominantemente la calidad (Pérez, 2010).

De acuerdo a reglamentos y declaratorias a nivel mundial, la educación superior se ha mantenido bajo constantes cambios debido al desarrollo tecnológico. Según lo expresado por la Declaración Mundial sobre la Educación Superior en el Siglo XXI, esta se fundamenta en la visión y acción para determinar su facultad de transformarse y enfocarse en la prosperidad, lo cual podría entenderse como un preámbulo a sensibilizar las posibles demandas del entorno (Diago, 2013).

Por ejemplo, en el Ecuador, la Constitución de la República garantiza el derecho a la educación, donde señala "que la educación es un derecho de las personas" (Asamblea Constituyente, 2009, p. 26) siendo un deber obligatorio e inexcusable para la sociedad y sus ciudadanos. En el mismo documento dice que "se garantizará la libertad de enseñanza, y de cátedra en la educación superior", (Asamblea Constituyente, 2009 p.
28) señalando "como finalidad la formación académica y profesional con visión científica y humanista; la investigación científica y tecnológica; la innovación, promoción, desarrollo y difusión de los saberes y las culturas; la construcción de soluciones para los problemas del país" (Asamblea Constituyente, 2009 p. 162).

Así también, en el artículo 351 del mismo documento, se determina que el Sistema de Educación Superior estará relacionado al Sistema Nacional de Educación y al Plan Nacional de Desarrollo, siendo la ley quien determinará los procedimientos de conexión entre Sistema de Educación Superior y la Función Ejecutiva. Es así como indica que está bajo "los principios de autonomía responsable, cogobierno, igualdad de oportunidades, calidad, pertinencia, integralidad, autodeterminación para la producción del pensamiento y conocimiento, en el marco del diálogo de saberes, pensamiento universal y producción científica tecnológica global" (Asamblea Constituyente, 2009 p. 162). En el artículo 352 se establece que las instituciones que conforman el Sistema de Educación Superior deberán estar debidamente acreditadas y evaluadas. Las Instituciones de Educación Superior (IES), sean públicas o privadas, sin fines de lucro, estarán tuteladas por tres organismos públicos: SENESCYT, CACES y CES, con potestad sobre la fundación, planificación, acreditación, regulación y coordinación interna del sistema, y la relación con los diferentes miembros con la Función Ejecutiva.

Para profundizar la información proporcionada cabe indicar que dentro de las IES se crean por ley, previa notificación obligatoria de las instituciones responsables. A su vez, los organismos encargados de la acreditación y aseguramiento de la calidad tienen la potestad de suspender a las IES. Reconociéndose el derecho a la autonomía, al ejercicio establecido de libertad de cátedra en la 
exploración a la verdad del conocimiento, sin limitaciones. De la misma manera, en este documento establece que la autonomía no libera de que "las instituciones del sistema de ser fiscalizadas, de la responsabilidad social, de la rendición de cuentas y de la participación activa en la planificación nacional" (Asamblea Nacional, 2010 p. 5).

Incluso, dentro de la Ley Orgánica de Educación Superior, se definen principios que garantizan el derecho a la formación superior de calidad, el acceso, continuación, movilidad y egresamiento sin discriminación, estableciendo lo que indica esta ley. En el artículo 1, "los organismos e instituciones que lo integran, determina los derechos, deberes y obligaciones de las personas naturales y jurídicas, y establece las respectivas sanciones por el incumplimiento de las disposiciones constitucionales y de ley", según lo establecido en la Constitución de la República (Asamblea Nacional, 2010, p.5) .

De lo expuesto, a través de la exigencia legal vigente, los docentes de las universidades en el Ecuador podrían verse expuestos a altos niveles de presión debido a la carga laboral demandante (docencia, investigación y gestión), en donde la dedicación horaria a docencia incluye: preparación de clases, seminarios y talleres; elaboración de material didáctico y calificación de pruebas, trabajos, deberes, participación en proyectos de ámbitos artísticos, sociales, orientación, adiestramiento y seguimiento los estudiantes del SNNA (Asamblea Nacional, 2010).

En cuanto a las actividades de gestión, este reglamento indica que comprende: " $\mathrm{Di}$ rección y gestión de los procesos de docencia e investigación en sus distintos niveles de organización académica e institucional, revisor de una revista indexada o arbitrada, o de una publicación revisada por pares" (Consejo Superior de la Pontificia Universidad Católica del Ecuador, 2017, p. 4). Es decir, lo que implica en la mayoría de los casos sobrecargan de trabajo al querer combinar todas estas exigencias al mismo tiempo, y, a su vez, energía física y mental que requieren las necesidades personales y familiares.

De lo expuesto, dentro de leyes, estatutos y ordenanzas, la educación en el país se encuentra encaminada a alcanzar reconocimiento a nivel internacional y también busca que se lleve a cabo un trabajo consistente en cuanto a parámetros de calidad y de esfuerzo continúo brindando educación superior que redunde en oportunidades para los estudiantes. En ese sentido, los docentes deben aprovechar coherentemente los recursos y los procesos a fin de perfilar carreras e instituciones con proyección y pertinencia, no solo dentro del aula, sino también fuera de ella. Por otro lado, busca ahondar en la gestión administrativa que incluye componentes de investigación y gestión que se consideran esenciales y demandantes dentro de su labor, como lo establece el documento "La calidad en la universidad ecuatoriana" donde indica textualmente que: "Requieren planificar el desarrollo institucional estratégicamente y evaluar sistemáticamente el trabajo universitario y sus resultados" (CONEA, 2003, p. 10).

La búsqueda de la calidad en la vida laboral, como parte de un enfoque personal o grupal, puede afectar a los trabajadores en la percepción del entorno, y puede derivar en sentimientos de satisfacción o frustración como un esquema significativo de la situación para el individuo. Tomando en cuenta dos aspectos primordiales como: "El clima organizacional y los factores psicológicos", el resultado del estudio ha reflejado también evidencias económicas positivas para las organizaciones, ya que repercuten directamente, estimando su magnitud como un ente dinámico por la continua interacción entre los actores que participan en este (Espinoza, 2014). 
El estudio de las enfermedades humanas relacionadas tanto con las épocas y cultura en las que se desarrollan, y condicionado a su tiempo biológico, social y personal del ser humano, indica que la afectación de la salud, respecto al entorno, debe "analizar las enfermedades como realidades biológicas cambiantes y sometidas a circunstancias ambientales determinadas, como fenómenos sociales establecidos por estructuras socioeconómicas e integradas en patrones socioculturales concretos y como vivencias personales existentes en cada situación histórica" (Rojas, 2004).

Los factores que se describen como causantes de estas enfermedades, son un determinante en la afectación de la salud humana, no solo en las enfermedades patológicas, sino también aquellas que, sin existir afectación física, tienen repercusiones en la salud humana. Es así, que las empresas miden la tasa de plusvalía en función del costo del trabajo, aplicando procesos que permiten la optimización de los tiempos del trabajador de taller o de oficina (Chesnais y Serfati, 1996).

De acuerdo con lo expuesto, se muestra que las crisis financieras, guerras y demás acontecimientos de alto impacto en la sociedad, han permitido que los grandes inversionistas tengan la oportunidad de acumular riquezas excesivas, contrario a cuando la economía se mantiene estable lo que infiere necesariamente en más exigencias laborales que pesan sobre los trabajadores. Principalmente, por la desvalorización del empleo, y la sobreexplotación laboral exigiendo mayor cantidad de tiempo en el trabajo, vacaciones pagas más cortas, al crear una idea del trabajo en la como dice el investigador Chesnais (1997): "Lo que puede decirse sobre el componente "moral e histórico" en la determinación de los salarios, vale también palabra por palabra para las condiciones en las que la fuerza de trabajo es explotada en los empleos" (Chesnais \& Serfati, 1996, p. 15).
Partiendo de la premisa de que el síndrome de burnout comienza desde la concepción que tiene una persona de sí misma y de quienes se interrelacionan directamente con ella, este estudio se origina desde la conciencia personal del reflejo de las emociones y de los escenarios de los que forma parte. Es así cómo se puede vislumbrar el hecho de la incidencia de esta "respuesta prolongada" a la sensación de estrés que se produce dentro de la educación por considerarse una "experiencia altamente emocional". Todo esto es una consecuencia ya que los educadores forman parte de un conjunto de expectativas creadas, tanto por la sociedad, como por el propio sistema educativo vigente en cada país, es decir, que existe una estrecha vinculación con lo que se espera a futuro de los ciudadanos y su progreso (Sauter et al., 2001 p. 74).

El trabajo, como tal es parte fundamental de la evolución y el desarrollo de las personas, ya que no solo les da un lugar en la sociedad, sino que también les permite ser parte protagónica de la prosperidad y desarrollo de los pueblos. De esta forma, se entiende al trabajo como inmanente de los seres humanos y su propósito en la vida; es así que los alcances del entorno dentro de la salud y bienestar son imperiosos. Por ello, se releva la importancia de la salud ocupacional y los riesgos psicosociales a los que está expuesto el trabajador, y cómo estos afectan la calidad de vida en relación directa con lo propuesto dentro de la pirámide de necesidades de Maslow, que derivan en enfermedades que lo pueden llevar a vivir situaciones de "estrés prolongado" que desencadene el síndrome de burnout (Freire et al., 2015).

Cabe destacar que un estudio sobre Inteligencia emocional y rasgos de personalidad destaca que el caso específico de los docentes al establecer dinámicas de trabajo colaborativo o de mayor participación de los alumnos con estrategias psicopedagógicas 
más eficientes. Esto permite que la actividad diaria no se torne monótona generando el sentido de agotamiento e insatisfacción que termina por desmoralizar y desemboca en problemas de salud física (Bedoya, Vega, Severiche y Meza, 2017).

Existan variables importantes al momento de hablar de este problema, tales como "competencia profesional", "autoconfianza", "apoyo social de los compañeros", "apoyo social del supervisor" y "conflicto del rol" entre muchos otros, dichas variables a su vez trascienden en el nivel de burnout que desarrollan los individuos (Gil-monte \& Peiró, 1999).

El rendimiento profesional es otro de los factores que se ven afectados cuando se presenta el "agotamiento" por parte de los docentes, entendiéndose su labor como altamente estresante desatendiendo las demandas percibido como ineficacia lo que redunda en una deficiente evaluación de la gestión realizada (Herman, Hickmon-Rosa, y Reinke, 2017).

Es de suma importancia estudiar la posibilidad de que exista un grado o nivel de estrés, representada por docentes de la carrera, respecto a las exigencias que presenta el trabajo en relación con los compromisos adquiridos acorde a la asignación horaria de docencia, gestión e investigación. En muchos casos, de acuerdo con la normativa NTP 349, se podrá adoptar medidas de protección para que este riesgo psicosocial no afecte mayormente a los docentes, relacionado con los niveles de ausentismo y deserción; así como también se pueda tomar en cuenta para establecer nuevos tipos de medición de cumplimiento de tareas a realizarse (Centro Nacional de Condiciones de Trabajo, 1999).

Por último, es importante destacar que la trasparencia de este estudio a posterior se podrá aplicar para diagnosticar la viabilidad del clima organizacional, permitiendo proporcionar herramientas para la evalua- ción de las relaciones de tipo interpersonal y la interacción entre los distintos actores. Los estudios realizados sobre el sindrome de burnout en docentes se centra en todos los niveles de estudio; sin embargo, son los docentes universitarios quienes en su mayoría reflejan la presión no solo de las demandas, sino también la que establece la globalización y subsecuentemente la necesidad de adaptarse a nuevos entornos y retos que posteriormente se convierten en "manifestaciones de un desequilibrio del organismo" (Barbosa, Muñoz y Rueda, 2009).

\section{Metodología}

El tipo de investigación es descriptiva y comprende la conceptualización teórica, la descripción, el análisis e interpretación de la naturaleza actual de la problemática planteada.

Se partió desde una investigación bibliográfica y de una encuesta realizada a un grupo de docentes. En esta encuesta se aplica un muestreo estadístico no probabilístico y de carácter intencional que se obtuvo de un diagnóstico que permitirá enfocarse en las conclusiones; dichas conclusiones se contrastan y verifican a través de la prueba chi cuadrado en relación con los datos cualitativos.

La muestra analizada es de 17 docentes de la Carrera de Finanzas y Auditoria, que actualmente se encuentran con una demanda de trabajo importante, y en su comportamiento reflejan manifestaciones como mal humor, cansancio, dolores musculares y otros padecimientos. La encuesta se realizó de forma anónima, para conservar la trasparencia de los datos obtenidos, esta contiene 12 preguntas estructuradas que se obtuvieron de un análisis minucioso de la bibliografía consultada, con el propósito de vincular la relación existente entre la demanda laboral y la sintomatología característica del síndrome burnout. 
La encuesta se aplicó a través de la plataforma Google Drive y evidenció la administración adecuada del tiempo dentro y fuera de la universidad (horas de gestión, docencia e investigación), estilo de vida, rasgos de personalidad, niveles de estrés, relaciones interpersonales, nivel académico y aspectos personales importantes tales como edad, género, estado civil actual o el número de hijos (Hernández, Fernández y Baptista, 2010; Alaminos y Castejón, 2006).

\section{Resultados}

El análisis de los resultados parte de la correlación simple de las preguntas planteadas al inicio de esta investigación, misma que, combinadas con datos relevantes como el sexo, la edad y el estado civil, nos permiten esclarecer las variables del problema indicado.

Las preguntas y su análisis son los siguientes:

¿Cuáles son los principales síntomas que afectan a los docentes de la carrera en relación con su falta de energía y a sus niveles de estrés?

Los pesos porcentuales en relación con la sintomatología se encuentran mayormente afectados por tensión muscular y dolor de cabeza con valores de 37,5\% y $31,25 \%$ correspondientemente. Esto, tomando en cuenta que los niveles de estrés se encuentran entre altos y medios con un $50 \%$ y $37,50 \%$, y correlacionado con la falta de energía con un 56,25\% (Ver Tabla 1).

Tabla 1. Relación entre el nivel de estrés y la sintomatología del docente de carrera

\begin{tabular}{|l|l|l|l|l|}
\hline \multicolumn{1}{|c|}{$\begin{array}{c}\text { Relación entre nivel de estrés } \\
\text { y la sintomatología }\end{array}$} & \multicolumn{1}{|c|}{ Alto } & \multicolumn{1}{c|}{ Medio } & \multicolumn{1}{c|}{ Bajo } & \multicolumn{1}{|c|}{ Total } \\
\hline Tensión muscular & $31,25 \%$ & $6,25 \%$ & $0,00 \%$ & $37,50 \%$ \\
\hline Dolor de cabeza & $12,50 \%$ & $18,75 \%$ & $0,00 \%$ & $31,25 \%$ \\
\hline Problemas digestivos & $6,25 \%$ & $12,50 \%$ & $0,00 \%$ & $18,75 \%$ \\
\hline Ninguna de las anteriores & $\mathbf{0 , 0 0 \%}$ & $\mathbf{0 , 0 0 \%}$ & $\mathbf{1 2 , 5 0 \%}$ & $12,50 \%$ \\
\hline Total & $\mathbf{5 0 , 0 0 \%}$ & $\mathbf{3 7 , 5 0 \%}$ & $\mathbf{1 2 , 5 0 \%}$ & $\mathbf{1 0 0 , 0 0 \%}$ \\
\hline
\end{tabular}

Fuente: Resultados de encuestas

Figura 1. Relación de sintomatología y el nivel de estrés. Fuente: Resultados de encuestas

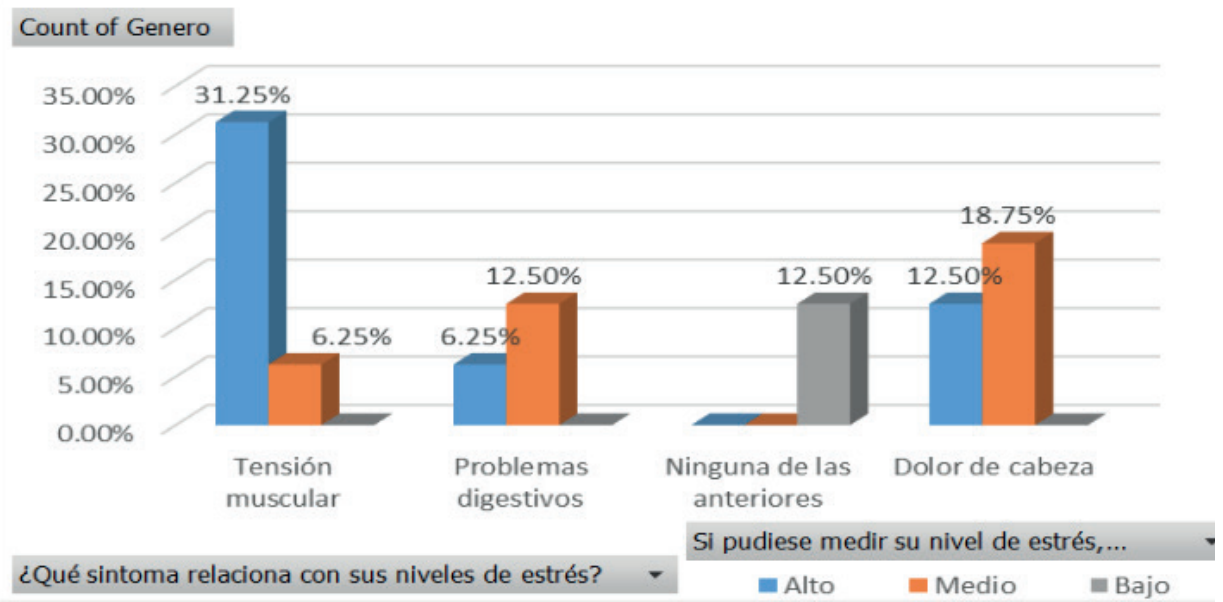


Aplicando la prueba de chi cuadrado a las variables cualitativas, con un estadístico de prueba de 15,9 y una cantidad de grados de libertad de 6, se mide la probabilidad de 0,01431 que determina la existencia afectación entre el nivel de estrés con la sintomatología de los docentes.

¿El clima organizacional se ve afectado por los niveles de estrés?

El clima organizacional se puede medir a través de la forma en la cual se interre- lacionan los individuos en su espacio de trabajo, en la muestra se señala que el vínculo únicamente es cordial y de respeto sin llegar a ser amigos con un 50\%; es decir, no se ven involucrados mayormente, lo cual no les permite apoyarse unos con otros en ámbitos laborales incluso personales, y pueden llegar a sobrecargarse en cuanto a sus emociones y esto derivar en otro tipo de afectaciones (ver tabla 2).

Tabla 2. Relación la falta de energía con el nivel de estrés

\begin{tabular}{|l|l|}
\hline \multicolumn{1}{|c|}{ Relación de falta de energía con el nivel de estrés } & \multicolumn{1}{|c|}{ Porcentaje } \\
\hline Sí & $\mathbf{5 6 , 2 5 \%}$ \\
\hline Alto & $25,00 \%$ \\
\hline Medio & $25,00 \%$ \\
\hline Bajo & $6,25 \%$ \\
\hline No & $\mathbf{4 3 , 7 5 \%}$ \\
\hline Alto & $25,00 \%$ \\
\hline Medio & $12,50 \%$ \\
\hline Bajo & $6,25 \%$ \\
\hline Total & $\mathbf{1 0 0 , 0 0 \%}$ \\
\hline
\end{tabular}

Fuente: Resultados de encuestas

Figura 2. Relación de la falta de energía y el nivel del estrés. Fuente: Resultados de encuestas

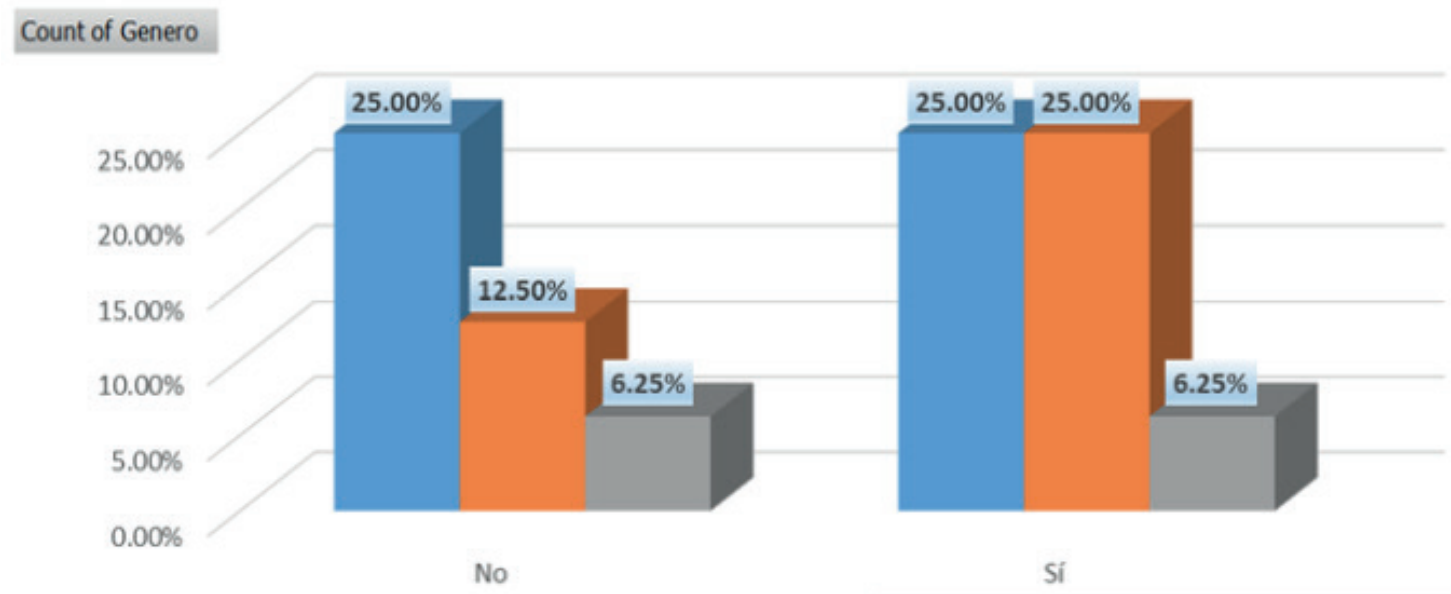


Aplicando la prueba de chi cuadrado a las variables cualitativas, con un estadístico de prueba de 0,42328, y una cantidad de grados de libertad de 2 , se mide la probabilidad de 0,8092558. La prueba de chi cuadrado establece que no existe una relación entre la falta de energía con el nivel de estrés de los docentes.
¿Qué género se ve mayormente afectado con frecuentes cambios de humor $y$ cuál es la tendencia en cuanto al humor?

De acuerdo a las respuestas, los docentes hombres se ven mayormente afectados representando un 37,50\%, con la ira (enojo e irritabilidad constantes); en relación con las mujeres un 6,25\% tiene tendencia a la ira (enojo e irritabilidad constantes), y el $25 \%$ no. (ver tabla 3 ).

Tabla 3. Relación con sus compañeros de trabajo

\begin{tabular}{|c|c|c|c|c|}
\hline $\begin{array}{l}\text { Género, cambio de humor } \\
\text { y tendencia en cambio de humor }\end{array}$ & Sí & No & A veces & Total \\
\hline Hombre & $37,50 \%$ & $12,50 \%$ & $18,75 \%$ & $68,75 \%$ \\
\hline Ira (enojo e irritabilidad constantes) & $18,75 \%$ & $0,00 \%$ & $18,75 \%$ & $37,50 \%$ \\
\hline Depresión (tristeza prolongada y pesimismo) & $18,75 \%$ & $0,00 \%$ & $0,00 \%$ & $18,75 \%$ \\
\hline Ninguna de las anteriores & $0,00 \%$ & $12,50 \%$ & $0,00 \%$ & $12,50 \%$ \\
\hline Mujer & $6,25 \%$ & $25,00 \%$ & $0,00 \%$ & $31,25 \%$ \\
\hline Depresión (tristeza prolongada y pesimismo) & $6,25 \%$ & $12,50 \%$ & $0,00 \%$ & $18,75 \%$ \\
\hline Ninguna de las anteriores & $0,00 \%$ & $12,50 \%$ & $0,00 \%$ & $12,50 \%$ \\
\hline Total & $43,75 \%$ & $37,50 \%$ & $18,75 \%$ & $100,00 \%$ \\
\hline
\end{tabular}

Fuente: Resultados de encuestas

Figura 3. Resultado de la relación con los compañeros. Fuente: Resultados de encuestas

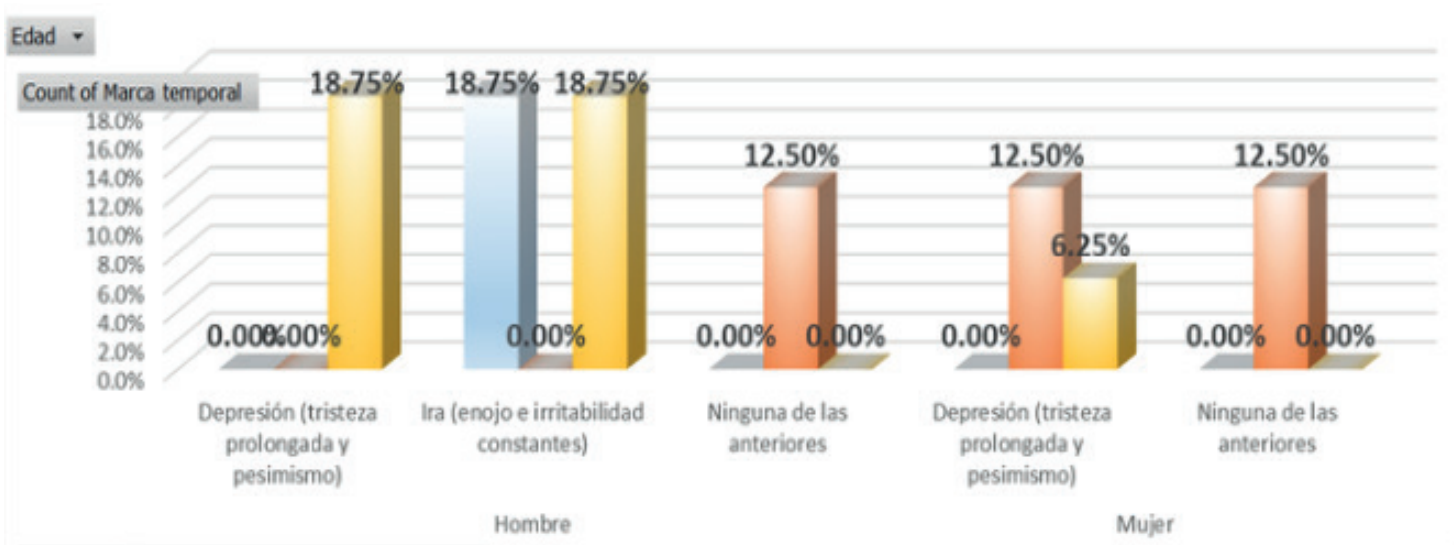

¿Qué tipo de afectación existe a nivel de la vida familiar, personal y de pareja en relación con el tiempo compartido y cómo lo califican?
En esta pregunta, se analiza la relación con el entorno fuera de su lugar de trabajo y se obtiene un porcentaje del $50 \%$ entre la evaluación de regular y malo. A su 
vez, se indica que el tiempo compartido tiene predominancia en $56,25 \%$ en "poco

a penas compartimos una comida juntos" (ver tabla 4).

Tabla 4. La calidad del tiempo que pasa con familia y amigos, y sus efectos en sus relaciones

\begin{tabular}{|l|l|l|l|l|l|}
\hline $\begin{array}{l}\text { Afectación del tiempo que se brinda con } \\
\text { la relación familiares y amigos }\end{array}$ & Excelente & Bueno & Regular & Malo & Total \\
\hline $\begin{array}{l}\text { Mucho, tengo tiempo para compartir con } \\
\text { mi familia y amigos }\end{array}$ & $12,50 \%$ & $0,00 \%$ & $0,00 \%$ & $0,00 \%$ & $12,50 \%$ \\
\hline $\begin{array}{l}\text { Poco, a penas compartimos una comida } \\
\text { juntos }\end{array}$ & $18,75 \%$ & $12,50 \%$ & $18,75 \%$ & $6,25 \%$ & $56,25 \%$ \\
\hline $\begin{array}{l}\text { Nada, cuando llego mi familia y/o ami- } \\
\text { gos ya están descansando }\end{array}$ & $0,00 \%$ & $6,25 \%$ & $\mathbf{6 , 2 5 \%}$ & $\mathbf{1 8 , 7 5 \%}$ & $\mathbf{3 1 , 2 5 \%}$ \\
\hline Total & $\mathbf{3 1 , 2 5 \%}$ & $\mathbf{1 8 , 7 5 \%}$ & $\mathbf{2 5 , 0 0 \%}$ & $\mathbf{2 5 , 0 0 \%}$ & $\mathbf{1 0 0 . 0 0 \%}$ \\
\hline
\end{tabular}

Fuente: Resultados de encuestas

Figura 4. Relación y el tiempo que comparte en familia y amigos. Fuente: Resultados de encuesta

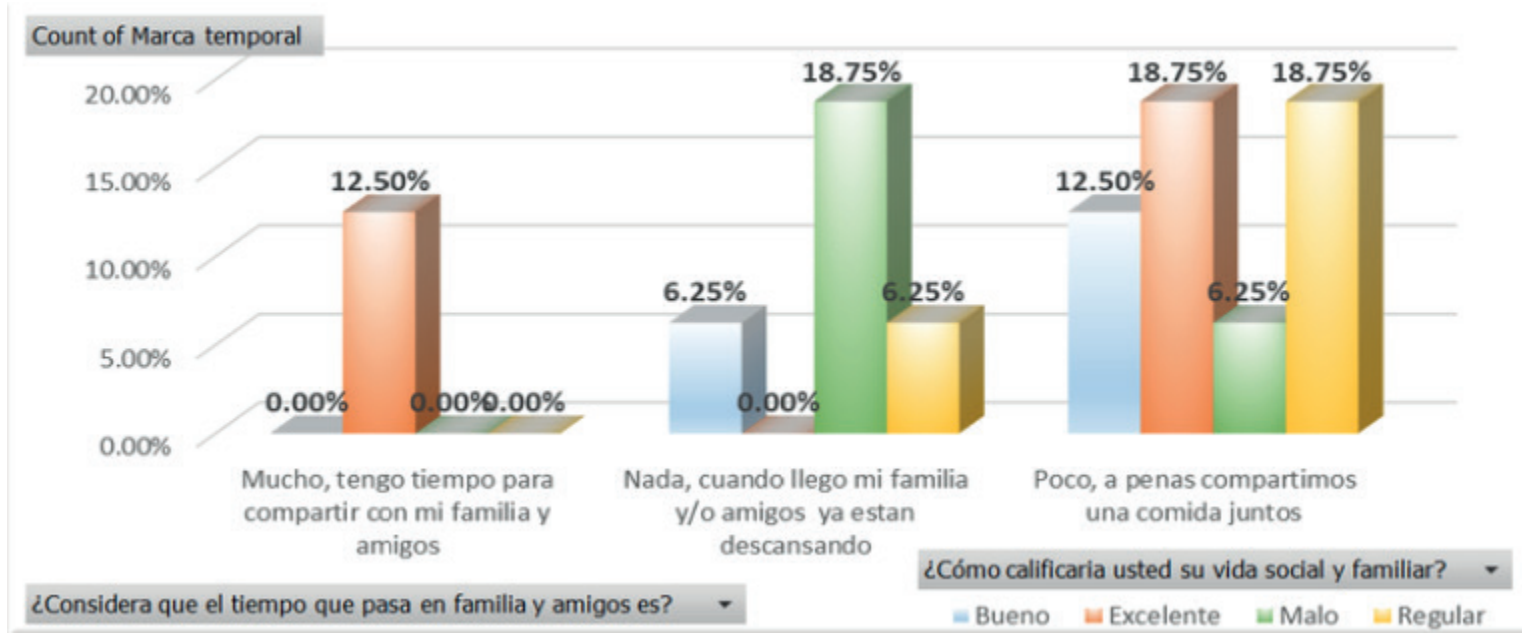

Aplicando la prueba de chi cuadrado a las variables cualitativas, con un estadístico de prueba de 6,5439, y una cantidad de grados de libertad de 6 , se mide la probabilidad de 0,350 , determinando que no existe una relación entre la calidad y el tiempo dado a relaciones sociales.

\section{¿Cuál es el indice de morbilidad?}

En esta pregunta busca analizar la frecuencia con la que se enferman en un periodo de tiempo alcanzando un valor de 37,5\% entre una vez al mes y 3 veces al mes. (Ver Tabla 5) 
Tabla 5. ¿Relación del índice de morbilidad?

\begin{tabular}{|l|l|}
\hline \multicolumn{1}{|c|}{ Frecuencia de enfermedades } & \multicolumn{1}{c|}{ Porcentaje } \\
\hline Tres veces al mes & $6,25 \%$ \\
\hline Una vez al mes & $31,25 \%$ \\
\hline Cada dos meses & $25,00 \%$ \\
\hline Dos veces al año & $25,00 \%$ \\
\hline Una vez al año & $12,50 \%$ \\
\hline Total & $\mathbf{1 0 0 , 0 0 \%}$ \\
\hline
\end{tabular}

Elaboración: Los autores

Figura 5. Análisis de índice de morbilidad. Fuente: Resultados de encuestas

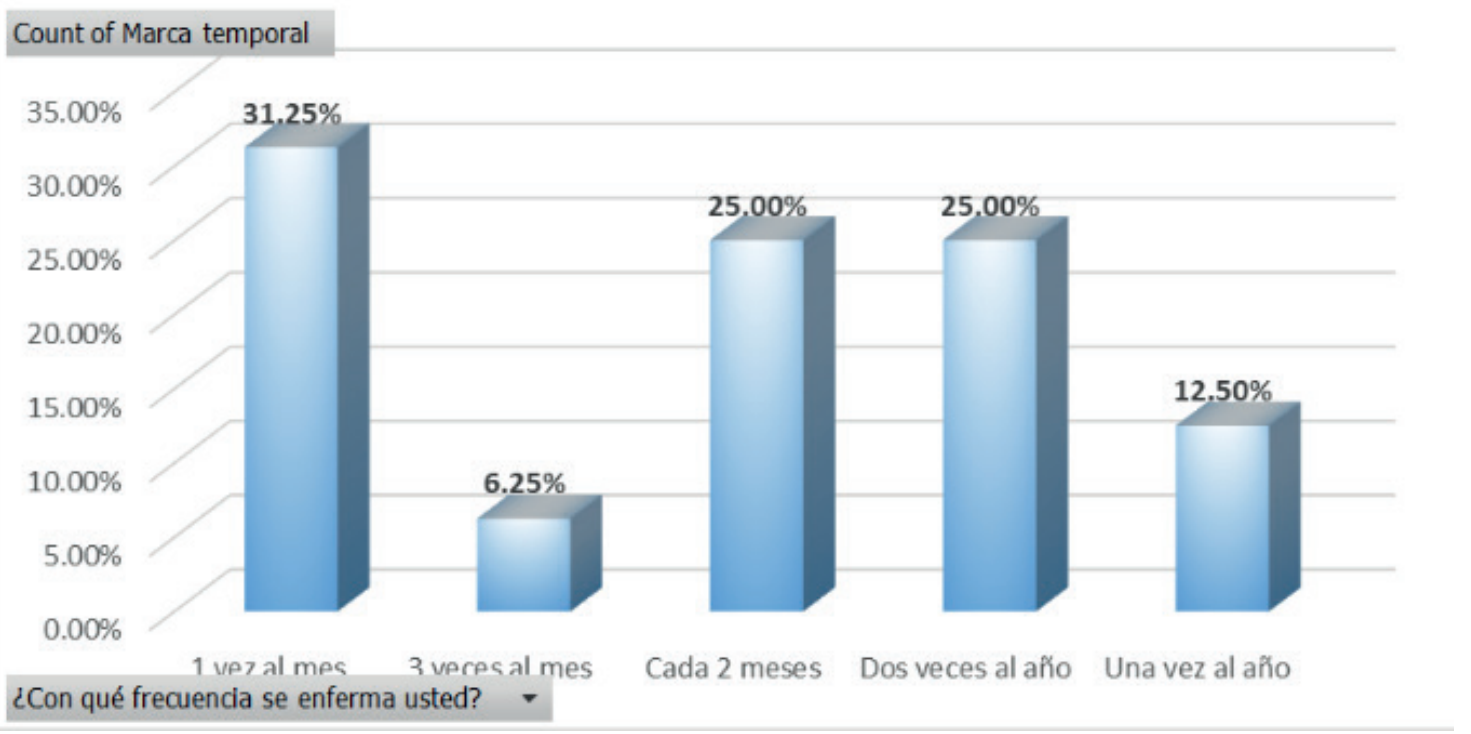

Para conocer la fragilidad individual a

Las respuestas indican que existe padecer el síndrome de burnout, se consultó una preeminencia de sedentarismo con un sobre el tipo de estilo de vida de vida de los $56,25 \%$ como lo indica la tabla 6. docentes.

Tabla 6. Relación de los docentes, ¿Considera que el tiempo que pasa en familia y amigos es?

\begin{tabular}{|l|l|}
\hline \multicolumn{1}{|c|}{ Tipos de estilos de vida } & \multicolumn{1}{c|}{ Porcentaje } \\
\hline Sedentario & $56,25 \%$ \\
\hline Atlético & $25,00 \%$ \\
\hline Saludable & $18,75 \%$ \\
\hline Total & $100,00 \%$ \\
\hline
\end{tabular}

Fuente: Resultados de encuestas 
Figura 6. Estilo de vida de los docentes. Fuente: Resultados de encuestas

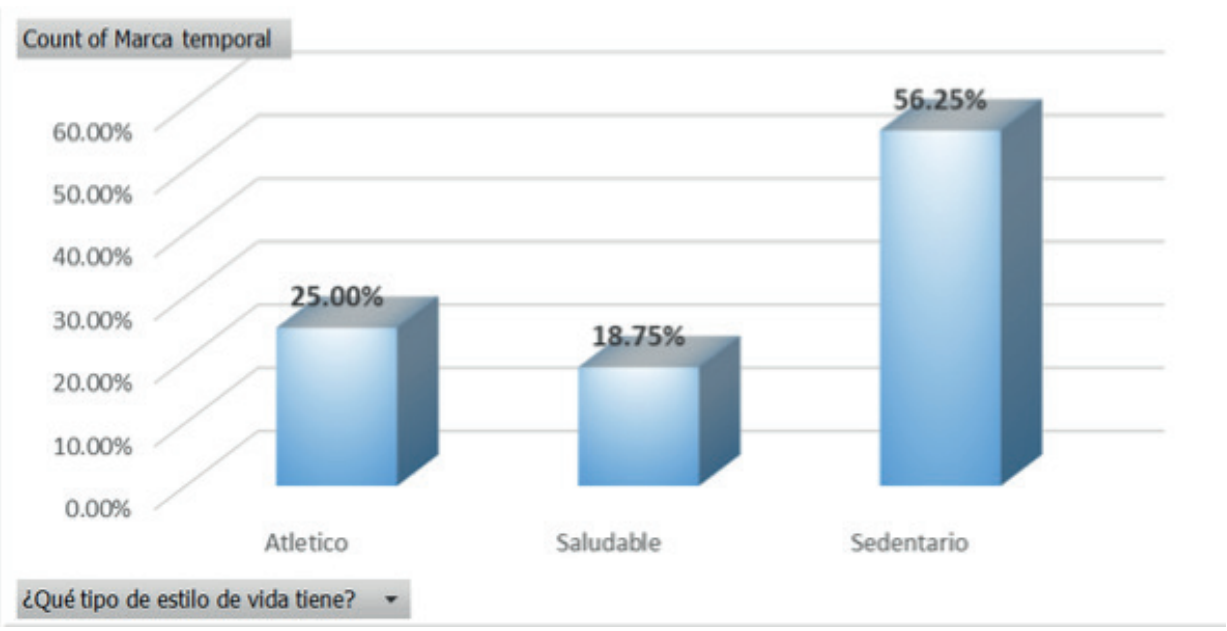

¿Cómo consideran los docentes sus rasgos de personalidad?

Las respuestas exponen que existe un predominio del sentido de responsabilidad y, a la vez, un neuroticismo implícito por la presión que puede derivar del cumplimiento de las labores curriculares y extracurriculares alcanzando un porcentaje de 31,25\% en cada uno de estos enunciados (ver tabla 7).

Tabla 7. Rasgos de personalidad de los Docentes

\begin{tabular}{|l|l|}
\hline \multicolumn{1}{|c|}{ Personalidad de los Docentes } & \multicolumn{1}{|c|}{ Porcentaje } \\
\hline Sedentario & $56,25 \%$ \\
\hline Atlético & $25,00 \%$ \\
\hline Saludable & $18,75 \%$ \\
\hline Total & $100,00 \%$ \\
\hline
\end{tabular}

Fuente: Resultados de encuestas

Figura 7. Rasgos de personalidad. Fuente: Resultados de encuestas

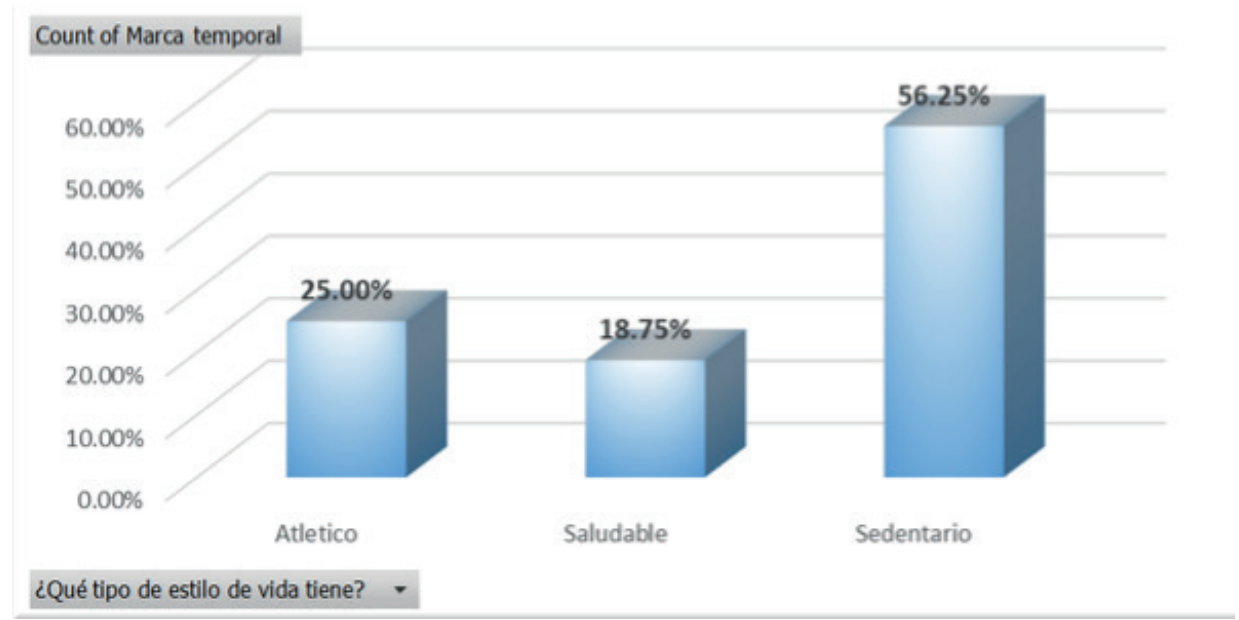


¿Cuántas horas dedica a docencia, gestión e investigación al cumplimiento de la normativa establecida?

El promedio de los datos obtenidos es de 46,31 horas combinadas entre las horas de docencia (calificar e impartir y preparar clases), actividades de gestión e investigación (ver tabla 8).

Tabla 8. Cuadro de indicadores generales de horas docencia, investigación y gestión de docentes

\begin{tabular}{|l|l|l|l|l|l|}
\hline Variables & $\begin{array}{c}\text { ¿Cuántas horas de- } \\
\text { dica a dictar clases } \\
\text { semanalmente? } \\
\text { Únicamente "dictar } \\
\text { clases" }\end{array}$ & $\begin{array}{c}\text { ¿Cuántas } \\
\text { horas dedica } \\
\text { a la prepara- } \\
\text { ción de clases } \\
\text { semanalmente? }\end{array}$ & $\begin{array}{c}\text { ¿Cuántas } \\
\text { horas dedi- } \\
\text { ca a gestión } \\
\text { semanalmente? }\end{array}$ & $\begin{array}{c}\text { ¿Cuántas } \\
\text { horas dedica a } \\
\text { investigación } \\
\text { semanalmente? }\end{array}$ & $\begin{array}{c}\text { Total, } \\
\text { Horas }\end{array}$ \\
\hline Promedio & 18,56 & 12,50 & 10,81 & 4,44 & 46,31 \\
\hline Máximo & 23 & 20 & 14 & 12 & 69 \\
\hline Mínimo & 12 & 12 & 8 & 0 & 32 \\
\hline Desviación & 4,05 & 2,00 & 2,64 & 4,19 & \\
\hline Varianza & 16,40 & 4,00 & 6,96 & 17,60 & \\
\hline
\end{tabular}

Fuente: Resultados de encuestas

\section{Discusión}

Esta investigación plantea como objetivo el estudio del síndrome de burnout en un grupo de docentes de la carrera de Contabilidad y Auditoría con un total entre 12 y 40 horas laborables a la semana, basándose principalmente en tres aspectos: docencia, investigación y gestión, mismos que son requeridos de acuerdo con lo que establece en el régimen académico vigente.

En relación con los resultados de la encuesta se señala lo siguiente:

- El nivel de estrés bordea el 50\%, de acuerdo con la sintomatología de tensión muscular y dolor de cabeza con un 69\%.

- Correlacionado con la falta de energía con un $56,25 \%$.

- El cambio de humor también sufre afectación con un 43,75\% (37,50\% en hombres).
- Se denotó que apenas el 31,25\% considera que la calidad de tiempo que pasa con la familia y amigos es excelente, el $50 \%$ se encuentra entre malo y regular.

- La morbilidad laboral indica que la ocurrencia de enfermedades se da entre cada dos meses y dos veces al año con un $50 \%$.

- El 56,25\% de los encuestados indican ser sedentarios.

- El promedio de tiempo de contacto con el docente es de 18 horas y el total entre docencia, investigación y gestión es de 46 horas semanales.

Luego de realizar el análisis correspondiente de los resultados obtenidos de las encuestas, se expusieron las variables que afectan directamente al docente y que, a su vez, repercuten en los individuos y los rasgos fundamentales en cuanto a salud, estilo de vida y personalidad. Esto nos permitió co- 
nocer más a fondo la percepción personal sobre cuestiones puntuales de su administración de tiempo y cómo lo dividen entre la vida laboral, personal y familiar.

Existen algunas herramientas para la recolección de los datos referentes al síndrome de burnout, todas se relacionan con las variables personales, organizacionales y ambientales que repercuten en forma directa en el cumplimiento de las funciones que llevan a cabo los trabajadores. Dentro de estas herramientas se encuentran el Maslach Burnout Inventory (MBI), Cuestionario para la Evaluación del Síndrome de Quemarse por el Trabajo (CESQT), Oldenburg Burnout Inventory (OLBI) y Copenhagen Burnout Inventory $(\mathrm{CBI})$, mismas que fueron la base para la elaboración de las preguntas que se plantearon en la encuesta (Serpa, 2013).

Las preguntas de la encuesta se enfocaron en el análisis de las horas trabajadas dentro y fuera de la universidad en el cumplimiento de la normativa legal vigente, y si es a consecuencia de las horas trabajadas que se producen cierta cantidad de síntomas o dolencias que sumados a la individualidad de los docentes encuestados llegan a resultar en el padecimiento de este riesgo psicosocial que se manifestaría en un detrimento o deterioro en su salud.

\section{Conclusiones}

- En la investigación bibliográfica se establecen las razones fundamentales por las que el estudio del síndrome de burnout, se debe aplicar preferentemente a aquellas ocupaciones que tienen una dedicación de tiempo importante al contacto con otras personas y esto implique una demanda de energía e interacción.

- La predisposición para que el síndrome de burnout aparezca es notorio dentro de nuestro medio social e institucional, por el nivel de exigencia en el desempeño dentro de las horas de gestión, docencia e investigación, incluyendo la necesidad de calidad y esmero que estas actividades implican. Por ello, los docentes son propensos a un desgaste intelectual, que inciden en la deficiencia laboral y personal.

- El síndrome de burnout se presenta como parte de un cambio sutil en el comportamiento de algunos de los encuestados, tomando en cuenta sus rasgos de personalidad y su estilo de vida. Esto se refleja en los datos sobre el tiempo dedicado a actividades de indole personal y a la administración del tiempo laboral, mismo que no se apega a las necesidades familiares $y$ de pareja.

- De los resultados de la investigación podemos concluir que, a mayor cantidad de horas totales de docencia, investigación y gestión de los docentes de la carrera, sus comportamientos manifiestan un cambio de temperamento (ira), probablemente provocado por la falta de tiempo para compartir con sus familias y amigos.

- Se puede concluir que el síndrome de burnout se desencadena como consecuencia de la individualidad de los docentes, donde se destaca su estilo de vida, sus rasgos de personalidad y el manejo de sus relaciones interpersonales, al responder con una mayor exigencia a las demandas del entorno.

- En el caso de la educación universitaria, existen lineamientos específicos sobre las actividades que deben cumplir y estos, a su vez, se encuentran normados legalmente dentro de la dedicación horaria de los docentes, mis- 
mas que pueden verse modificadas e incluso incrementadas con el objetivo de cumplir indicadores altos de calidad para mantener el estatus existente dentro de las IES.

- La prueba del chi cuadrado de datos cualitativos aplicada para la validación de la información obtenida en la relación de las variables del estrés con la sintomatología determinaron que existe una correspondencia directa y concreta respecto al nivel de estrés presentado por los docentes encuestados.

\section{Referencias}

Alaminos Chica, A., y Castejón Costa, J. (2006). El muestreo en la investigación social. Elaboración, análisis e interpretación de encuestas, cuestionarios y escalas de opinión. Alicante. Retrieved from https://rua.ua.es/dspace/bitstream/10045/20331/1/Elaboración, análisis e interpretación.pdf\#page $=41$

Arquero, J. L., y Donoso, J. A. (2013). Docencia, investigación y burnout: el síndrome del quemado en profesores universitarios de Contabilidad. Revista de Contabilidad, 16(2), 94-105. https://doi.org/10.1016/j. rcsar.2013.04.001

Asamblea Constituyente. (2009). Constitución del Ecuador. Registro Oficial, (20 de Octubre), 173. https://doi.org/10.1017/ CB09781107415324.004

Asamblea Nacional. Ley Orgánica de Educación Superior del Ecuador, Registro Oficial - Órgano del Gobierno del Ecuador \& (2010).

Asamblea Nacional. Ley Orgánica De Educación Superior Ecuador, Ley Orgánica de Educación Superior del Ecuador \& (2010).

Barbosa, Lucia; Muñoz, Maria; Rueda, P. S. K. (2009). Síndrome de burnout y estrategias de afrontamiento en docentes universitarios. Revista Iberoamericana De Psicología: Ciencia Y Tecnología 2(1): 21-
30, 2009 Sindrome, 1(1), 21-30. Retrieved from http://www.scielo.org.co/pdf/psdc/ n26/n26a03

Bedoya, E. A., Vega, N. E., Severiche, C. A., \& Meza, M. J. (2017). Síndrome de Quemado (Burnout) en Docentes Universitarios: El Caso de un Centro de Estudios del Caribe Colombiano. Formación Universitaria, 10(6), 51-58. https://doi.org/10.4067/S071850062017000600006

Carlin M, \& Garcés E. (2010). El síndrome de burnout: Evolución histórica desde el contexto laboral al ámbito deportivo. Rev Anales de Psicología [revista en Internet] 2010 [acceso 26 de octubre de 2017]; 26(12) : [169-180], 26, 169-180. Retrieved from http://www.um.es/analesps/v26/ v26_1/20-26_1.pdf

Carlotto, Mary Sandra, Nakamura Antonieta Pepe, C. S. G. (2006). Sindrome de Burnout em estudantes universitários da área da saúde, 57-62.

Centro Nacional de Condiciones de Trabajo. Prevención del estrés: intervención sobre el individuo, Ministerio de Trabajo e Inmigración \& (1999).

Chesnais, F., y Serfati, C. (1996). La caracterización del capitalismo a fines del siglo XX. Herramienta.

CONEA. (2003). La calidad en la Universidad Ecuatoriana.

Consejo Superior de la Pontificia Universidad Católica del Ecuador. PONTIFICIA UNIVERSIDAD CATÓLICA DEL ECUADOR CONSEJO SUPERIOR REGLAMENTO GENERAL DE PERSONAL ACADÉMICO Y ESCALAFÓN DE LA PONTIFICIA UNIVERSIDAD CATÓLICA DEL ECUADOR Quito, febrero de 2017 (2017). Retrieved from https://www.puce.edu. ec/intranet/documentos/Reglamentos/ PUCE-REGLAMENTO-GENERAL-DE-PERSONAL-ACADEMICO-Y-ESCALAFON-DE-LAPUCE-APROBADO-POR-CS.pdf

Diago, F. (2013). De la visión a la acción . Declaración Mundial sobre la Educación Superior 1 From vision to action. World Declaration on Higher Education. Punto de Vista, 6, 173-183. 
Espinoza Díaz, I. M. (2014). Calidad de Vida y Salud Ocupacional en Docentes : efectos del clima psicosocial, la personalidad y el Síndrome de Quemado en el Trabajo. UNIVERSITAT ROVIRA I VIRGILI, 1-157.

Freire, M. A., Oliveira, E. N., Neto, F. R. G. X., Lopes, R. E., Ferreira, A. G. N., \& Gomes, B. V. (2015). Síndrome de Burnout: un estudio con profesores. Salud de Los Trabajadores, 23(1), 19-27.

Gil-monte, P. R., y Peiró, M. J. (1999). Perspectivas teóricas y modelos interpretativos para el estudio del síndrome de quemarse por el trabajo. Anales de Psicologia, 15(2), 261268

Granados, L., et. al. (2019). ¿Predicen los factores de depresión, ansiedad y estrés la dimensión de la despersonalización y la baja realización personal en el profesorado? European Journal of Child Development, Education and Psychopathology, 7(1), 83. https://doi.org/10.30552/ejpad.v7i1.91

Herman, K. C., Hickmon-Rosa, J., y Reinke, W. M. (2017). Empirically Derived Profiles of Teacher Stress, Burnout, Self-Efficacy, and Coping and Associated Student Outcomes. Journal of Positive Behavior Interventions, 2, 109830071773206. https://doi. org/10.1177/1098300717732066

Hernandez, R., Fernandez-Collado, C., y Baptista, P. (2010). Metodología de la Investigación (Cuarta Edi).

Murphy, J., \& Adams, J. E. (1998). Reforming America 's schools 1980-2000. Journal of Educational Administration, 36(5), 426-444.

Pérez, Á. (2010). Nuevas exigencias y escenarios para la profesión docente en la era de la información y de la incertidumbre Intro- duction: New demands and scenarios for the teaching profession in the age of information and uncertainty. Revista Interuniversitaria de Formacion Del Profesorado, 24, 17-36.

Perez Aranda, G. I., Angulo Gutiérrez, A. K., Cruz Vela, A. G., \& Estrada Carmona, S. (2018). Síndrome de burnout en el profesorado de la Universidad Autónoma de Campeche (México). Apuntes de Psicología, 36, 129-134.

Rojas, F. (2004). El componente social de la salud pública en el siglo XXI. Revista Cubana de Salud Pública, 30. Retrieved from http:// scielo.sld.cu/scielo.php?pid=S0864$34662004000300008 \&$ script $=$ sci_. arttext\%5Cnhttp://redalyc.uaemex.mx/ src/inicio/ArtPdfRed.jsp?iCve $=21430308$

Romero, A., Pimienta, I., Ramos, M., Sanchez, A., y González, R. (2019). Niveles de síndrome de burnout en docentes de una universidad ecuatoriana. Revista Dilemas Contemporáneos: Educación, Política y Valores, 8(5), 55. Retrieved from http://www. dilemascontemporaneoseducacionpoliticayvalores.com/

Sauter, S. L., Murphy, L. R., Hurrell, J. J., y Lennart, L. (2001). Factores psicosociales y de organizacion. Enciclopédia de Salud e Seguridad En El Trabajo, (1), 34.2-34.3. Retrieved from http://www.insht.es/InshtWeb/ Contenidos/Documentacion/TextosOnline/EnciclopediaOIT/tomo2/34.pdf

Serpa, A. V. (2013). Carga Mental y Sintomas/Signos del Sindrome de Burnout en Docentes a Tiempo Completo de Universidad Privada De Quito. 